\title{
Retrospective Correction of the Heel Effect in Hand Radiographs
}

\author{
G. Behiels, F. Maes`, D. Vandermeulen, and P. Suetens \\ Katholieke Universiteit Leuven \\ Faculties of Medicine and Engineering \\ Medical Image Computing (Radiology - ESAT/PSI) \\ University Hospital Gasthuisberg, Herestraat 49, B-3000 Leuven, Belgium \\ Gert.Behiels@uz . kuleuven.ac. be
}

\begin{abstract}
A method for retrospective correction of intensity inhomogeneities induced by the heel effect in digital radiographs is presented. The method is based on a mathematical model for the heel effect derived from the acquisition geometry. The model parameters are estimated by fitting the model to the image intensity data in the background or direct exposure area only where the heel effect is directly measurable, while the correction is then applied to the whole image. The method iterates between background segmentation and heel effect correction until convergence. We illustrate the performance of the method on flat field and phantom images and demonstrate its robustness on a database of 137 diagnostic hand radiographs.
\end{abstract}

\section{Introduction}

Digital radiography offers the possibility for computer aided diagnosis and quantitative analysis using image processing techniques such as segmentation $[2,3,4,8]$. But intensity-based segmentation of digital radiographs is hindered by intensity inhomogeneities inherent to the imaging process as illustrated by the hand radiograph shown in Fig. 2a: the background at the left side of the image is clearly brighter than at the right side. This phenomenon can be largely attributed to non-uniform X-ray exposure or heel effect. Although the spatially smoothly varying intensity inhomogeneity induced by the heel effect is easily corrected for by the human visual perception system, its presence complicates the use of automatic processing techniques because the brightness of an object within the image is position dependent. Intensity rectification based on calibration images is not feasible in practice because the image acquisition parameters that affect intensity inhomogeneity, such as the positioning of the recording device relative to the X-ray source, may vary from image to image and can not be recovered from the acquired image at read out.

In this paper, we present a fully automated retrospective method for intensity inhomogeneity correction of digital radiographs based on a mathematical model

\footnotetext{
* Frederik Maes is Postdoctoral Fellow of the Fund for Scientific Research - Flanders (FWO-Vlaanderen, Belgium).
} 


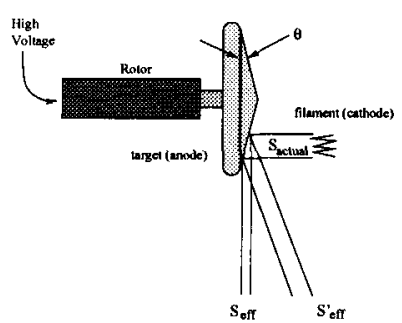

(a)

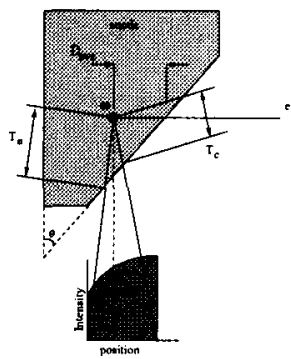

(b)

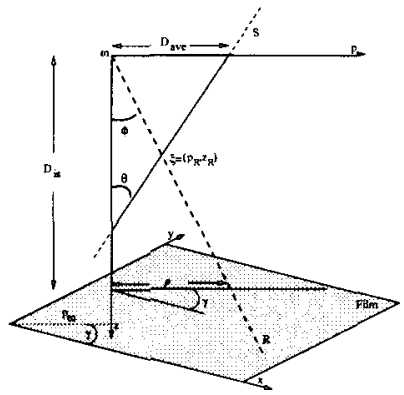

(c)

Fig. 1. (a)-(b) Schematic side views of an x-ray tube. The anode angle allows the use of a large focal spot $\left(S_{\text {actual }}\right)$ for heat-loading considerations and a small projected focal spot $\left(S_{\text {eff }}\right)$. X-rays are emitted at an average depth $D_{\text {ave }}$; the path length on the anode side $T_{\mathrm{a}}>T_{\mathrm{c}}$ is larger and causes a reduction in intensity. (c) X-ray coordinate system where the X-ray originates at position $(0,0)$ and travels along $R$ to the film at position $\left(p, D_{i s}\right)$.

for the heel effect derived from the acquisition geometry. Because the heel effect is only directly measurable in the direct exposure area, we first extract the image background and estimate the parameters of the model by fitting it to the image intensity in this region only. Inhomogeneity correction is then applied to the whole image, a new background region is extracted from the corrected data and the model parameters are re-estimated. This is repeated until no significant changes in background and parameter estimation occur. We demonstrate the performance of the method on a database of hand radiographs that were acquired for bone age determination.

\section{Modeling the Heel Effect}

The heel effect can be understood from the construction of the X-ray tube as schematically depicted in Fig. 1. Electrons originating from the cathode are attracted by the positively charged anode. For better heat dissipation, the anode rotates and is inclined by a small anode angle $\theta$ to enlarge the area $S_{\text {actual }}$ that is bombarded by electrons while keeping the size of the focal spot $S_{\text {eff }}$ fairly small. The X-rays, which can be thought of to originate from a point source $\omega$ at a depth $D_{\text {ave }}$ below the anode surface $S$, are therefore attenuated more by the anode material at the anode side than at the cathode side of the beam $\left(T_{a}>T_{c}\right)$. This results in a non-uniform $\mathrm{X}$-ray exposure of the imaging field, which explains the inhomogeneity of the background in the image of Fig. 2a.

A mathematical model for the heel effect can be derived from the simplified one-dimensional model of the anode and beam geometry depicted in Fig. 1c [5]. In the coordinate system $(p, z)$, with $p$ along the anode-cathode axis and $z$ along the vertical direction, the ray $R$ at an angle $\phi$ from the vertical within the plane 
$(\omega, S)$ originates at $\omega(0,0)$ and hits the recording device at point $\left(p, D_{\text {is }}\right)$ with $D_{\text {is }}$ the distance between the X-ray source and the recording device and $\tan \phi=$ $p / D_{\text {is }}$. The distance $r$ traveled by $R$ through the anode is $r=|\xi-\omega|=\sqrt{p_{R}^{2}+z_{R}^{2}}$ with $\xi\left(p_{R}, z_{R}\right)$ the intersection of $R$ with $S$. Solving the system of equations

$$
\left\{\begin{array}{l}
S: p_{R}=D_{\text {ave }}-z_{R} \cdot \tan \theta \\
R: p_{R}=z_{R} \cdot \tan \phi
\end{array}\right.
$$

yields:

$$
r(p)=D_{\text {ave }} \frac{\cos \theta}{\sin (\phi+\theta)}=D_{\text {ave }} \frac{\sqrt{1+\left(\frac{p}{D_{\text {is }}}\right)^{2}}}{\tan \theta+\frac{p}{D_{\text {is }}}}
$$

The incident radiation along the anode-cathode axis is therefore modeled by

$$
M(p, \alpha)=I_{0} \cdot e^{-\mu \cdot r \cdot\left(p-p_{\omega}\right)}
$$

with $\mu$ the attenuation coefficient of the anode material, $I_{0}$ the radiation originating at $\omega, p_{\omega}$ a parameter introduced to account for the fact that the position of the source relative to the recording device is unknown, and $\alpha$ the 5 model parameters $\left\{I_{0}, \mu . D_{\text {ave }}, \theta, D_{\text {is }}, p_{\omega}\right\}$. The model predicts that the heel effect behaves exponentially along the anode-cathode axis and assumes that it is constant perpendicular to this axis. This is justified by flat fieid exposure experiments which show that the difference in intensity perpendicular to the anode-cathode axis is relatively small compared to the intensity differences along this axis (see Fig. 3).

\section{Image Partitioning}

A typical hand radiograph, as shown in Fig. 2a, consists of three regions: collimation area, direct exposure area and diagnostic regions. Because the heel effect is directly measurable in the direct exposure area only, we first partition the image in order to estimate the parameters of model (1) from this region only. We do this by first extracting the collimation area and then searching the direct exposure area, the remaining areas being diagnostic regions.

We find the boundaries of the collimation area using the Hough transform [6], assuming that these are rectilinear edges as is the case for all hand radiographs in our database. To make this approach more robust, the contributions of each image point to the Hough accumulator are weighted by its gradient magnitude [1] and, for each point, only the lines whose direction is within 10 degrees from the normal to the local gradient direction are considered [7]. The 4 most salient points in Hough space that represent a quadragon with inner angles between 80 and 100 degrees are selected as candidate boundaries of the collimation area. Because not all 4 collimation boundaries are allways present in the image, candidate boundaries along which the image intensity differs from the intensity expected for the collimation region are rejected. A typical result is shown in Fig. 2a.

To extract the direct exposure or background region $B$, a seed fill algorithm is used that starts from the boundary of the collimation region as determined in 


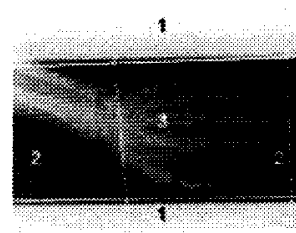

(a)

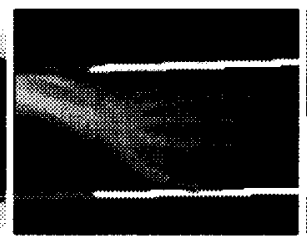

(b)

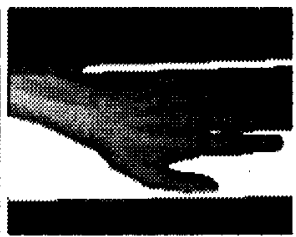

(c)

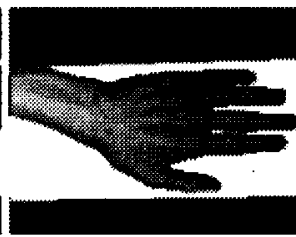

(d)

Fig. 2. (a) Typical hand radiograph with (1) collimation area, (2) direct exposure area, (3) diagnostic areas. The detected collimator edges are displayed as solid white lines and the rejected boundaries as white dotted lines. (b) Seed points for background region growing. (c,d) Intermediate and final stages of background region growing.

the previous step. Appropriate seed points for $B$ are found by considering a small band along each of the collimator edges and retaining all pixels whose intensity is smaller than the mean of the band. This approach avoids choosing pixels that belong to the diagnostic region as candidate seed pixels. $B$ is then grown by considering each 8-connected neighbor $n$ of each pixel $b$ in $B$ and adding $n$ to $B$ if the intensity difference between $b$ and $n$ is smaller than some fixed threshold. A few snapshots of the progress are shown in Fig. 2b-d.

\section{Heel Effect Estimation}

The orientation $\gamma$ of the anode-cathode axis and the model parameters $\alpha$ have to be determined such that (1) best fits the image intensity data $N(x, y)$ within the direct exposure area $B$, with $x$ and $y$ the image coordinates as defined in Fig. 1c. Assuming that $\gamma$ is known, the average image profile $P_{\gamma}(p)$ along this direction in the direct exposure region $B$ is given by

$$
P_{\gamma}(p)=\langle N(x, y)\rangle_{(x, y) \in B \mid x \cdot \cos \gamma+y \cdot \sin \gamma=p}
$$

with $\langle\cdot\rangle$ the averaging operator. We find the optimal model parameters $\alpha^{*}$ by a least square fit of the expected profile $M(p, \alpha)$ to the measured profile $P_{\gamma}(p)$ :

$$
\alpha^{*}(\gamma)=\arg \min _{\alpha}\left\|P_{\gamma}(p)-M(p, \alpha)\right\|
$$

The fitted one-dimensional model $M\left(p, \alpha^{*}(\gamma)\right)$ is then back projected perpendicular to the projection axis $\gamma$ to obtain a reconstruction $R\left(x, y, \gamma, \alpha^{*}(\gamma)\right)$ for the whole image:

$$
R\left(x, y, \gamma, \alpha^{*}(\gamma)\right)=M\left(x \cdot \cos \gamma+y \cdot \sin \gamma, \alpha^{*}(\gamma)\right)
$$

The orientation $\gamma$ is then determined such that this reconstruction best fits the actual image data within the direct exposure region:

$$
\gamma^{*}=\arg \min _{\gamma}\left\|N(x, y)-R\left(x, y, \gamma, \alpha^{*}(\gamma)\right)\right\|_{(x, y) \in B}
$$


or

$$
\gamma^{*}=\arg \min _{\gamma}\left\|\frac{N(x, y)}{R\left(x, y, \gamma, \alpha^{*}(\gamma)\right)}-1\right\|_{(x, y) \in B}
$$

depending on whether we wish to use additive or multiplicative correction. The estimated heel effect is $R\left(x, y, \gamma^{*}, \alpha^{*}\left(\gamma^{*}\right)\right)$ and the corrected image is respectively

$$
\hat{N}(x, y)=N(x, y)-R\left(x, y, \gamma^{*}, \alpha^{*}\left(\gamma^{*}\right)\right)
$$

or

$$
\hat{N}(x, y)=\frac{N(x, y)}{R\left(x, y, \gamma^{*}, \alpha^{*}\left(\gamma^{*}\right)\right)} .
$$

The optimal parameters $\alpha^{*}$ are found by multidimensional downhill simplex search [9], starting from initial values

$$
I_{0}=\max _{p}\left(P_{\gamma}(p)\right), \mu . D_{\text {ave }}=1, \theta=15 \mathrm{deg}, D_{\mathrm{is}}=1 \mathrm{~m}, p_{\omega}=0.15 \mathrm{~m}
$$

In our setup the anode-cathode axis is allways parallel to the image edges or the collimation edges. $\gamma^{*}$ is therefore found by exhaustive search over these 8 orientations only, which drastically reduces computation time.

After inhomogeneity correction of the image using (4) or (5), the direct exposure area $B$ is updated by thresholding, using a threshold derived from the histogram of the corrected image intensities $\hat{N}$. Keeping the previously determined anode-cathode orientation $\gamma$, new values for the optimal model parameters $\alpha^{*}$ are determined using (2) taking the newly selected direct exposure region into account. We thus iterate three or four times between background segmentation and heel effect correction until convergence.

\section{Results}

In all experiments additive correction using (3) and (4) was used. The model (1) was verified using a flat field image and an image of a hand phantom (Fig. 3 ). The heel effect is clearly visible in the flat field image by inspection of intensity traces along both image axes, showing a smooth degradation along the anode-cathode axis and an almost constant behavior perpendicular to this axis. A similar pattern is visible in the traces of the phantom image. Inspection of the traces of the corrected images shows that most of the background intensity variation is indeed eliminated using model (1). Physical models of X-ray production, recording and read-out predict a Gaussian distribution for the intensity of background pixels. This is not the case for the original flat field and phantom images (see histograms of Fig. 3a,d). However, the distribution of the background pixels of the corrected images is almost perfectly Gaussian (Fig. 3c,f), which is another indication that our model performs very well.

The method was evaluated on 137 digital hand radiographs, recorded with Agfa ADC cassettes and Agfa ADC-MD10 \& ADC-MD30 imaging plates and irradiated by X-ray tubes Philips SRM 0612 - ROT 500 or Siemens Bi 125/40 RL. 
(a)
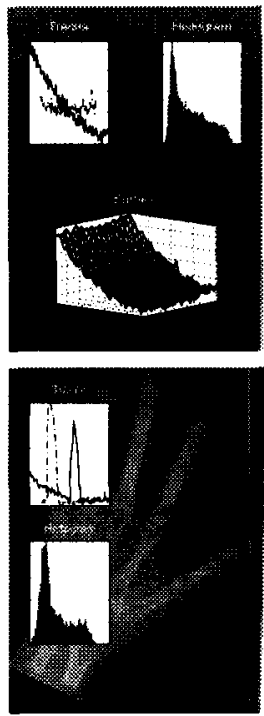

(d) (b)
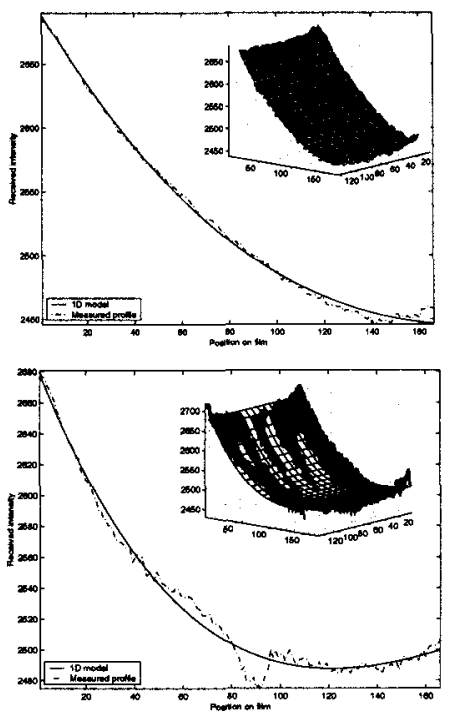

(e) (c)
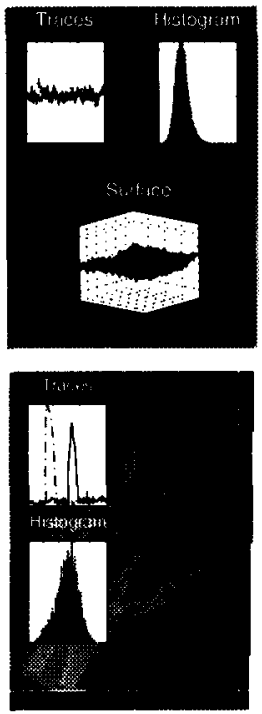

(f)

Fig. 3. (a) Flat field image (background) rendered as a surface (bottom) with histogram (top-right) and traces (top-left) along the anode-cathode axis (long solid line) and perpendicular to this axis (shorter dotted line). (b) Average projected data of the flat field (dotted line) and the fitted model (solid line). The sub-image contains back projected data (white grid) on top of the rendered background. (c) Same as (a) for the corrected image. (d) Same as (a) for the phantom image without the background surface and traces taken along the white lines. (e) Same as (b) for the phantom image. (f) Same as (d) for the corrected phantom image.

The images were downsampled by a factor 4 in each direcion to about $512 \times 512$ pixels. Visual inspection showed that the image partitioning algorithm was able to give very good estimates of the direct exposure, collimation and diagnostic areas for all images in our database, but erroneously includes some pixels of the soft tissue in the background region. Some results of the intensity correction are shown in Fig. 4. In the first image, the heel effect is clearly visible, while in the second example its presence can be detected from the increased background intensity at the bottom right of the images. For both cases the heel effect smears out the histogram of image background intensities, while after correction the background histogram is Gaussian distributed as it should. Specifying a histogram-derived threshold is sufficient to properly segment the hand after correction (top-right of each image), which is not possible for the original images due to the intensity overlap of diagnostic and background regions. Average computation time on a Pentium III $800 \mathrm{MHz}$ processor was 3 seconds, including image partitioning.

The possibly disturbing effect of introducing a hand in the image, thus including some soft tissue pixels coming from the partitioning algorithm and de- 

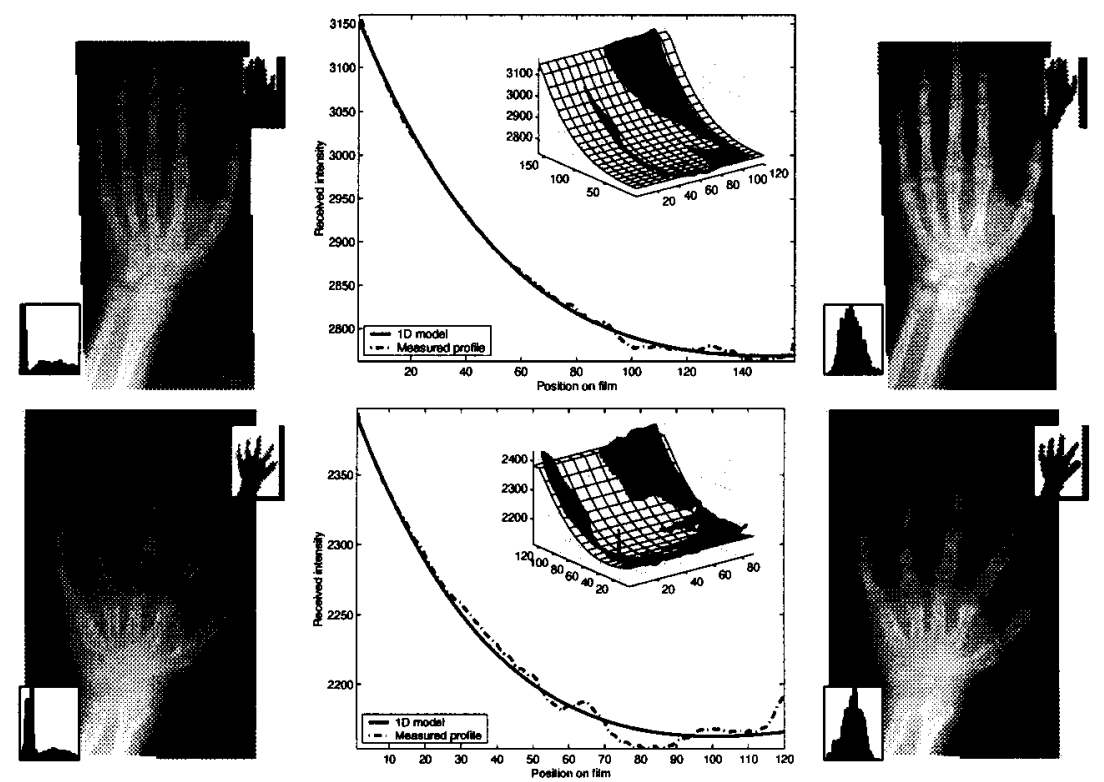

Fig. 4. Left column: original images with corresponding background histogram and histogram based background segmentation. Middle column: one-dimensional projection and back projected model fitted to the image data. Right column: corrected images with corresponding background histogram and histogram based background segmentation.

creasing the robustness of the estimated mean of the heel effect profile of (4), was examined by correcting the flat field image $N_{\text {ff }}$ once with parameters computed from the flat field image itself $R_{\mathrm{ff}}$ and once with parameters computed from the phantom image $R_{\mathrm{ph}}$. The reconstruction errors $\epsilon_{\mathrm{ff}, \mathrm{ph}}=N_{\mathrm{ff}}-R_{\mathrm{ff}, \mathrm{ph}}$ are very similar for both cases: $\langle\epsilon\rangle_{\mathrm{ff}}=-3.1, \sigma_{\epsilon}=12.4$, range $(-46.7,60.8)$ and $\langle\epsilon\rangle_{\mathrm{ph}}=-5.5, \sigma_{\epsilon}=27.1$, range $(-63.9,49.3)$ respectively, while the dynamic range of $N$ was 800 . This indicates that the algorithm can cope well with the presence of diagnostic regions when estimating the background inhomogeneity.

\section{Discussion}

We presented a fully automated method for heel effect correction of digital radiographs. We demonstrated the reliability of the method on a database of hand radiographs from which we could segment the hand very accurately by simple thresholding after inhomogeneity correction. The method is fast and reliable enough to be used for standardized image display on diagnostic workstations. It can also provide segmentation techniques with properly normalized images whose relative intensity differences are not disturbed by image inhomogeneities. We are currently investigating how this affects the specificity of intensity models constructed for Active Shape Model-based segmentation of the hand bones [2,3]. 
Our correction approach is based on a specific mathematical model for the heel effect derived from the acquisition geometry. While more general polygonal or spline-based models are commonly used in similar image rectification problems such as MR bias field correction [10], our method has the advantage to exploit knowledge about physical aspects of the imaging process. This reduces the number of degrees of freedom in the model while maintaining sufficient flexibility and makes the model fitting more robust, for instance to errors in the initial background region extraction.

The relatively simple theoretical model (1) is able to correct most of the variation present in the direct exposure area of the images that can be attributed to the heel effect. Nevertheless, we found that for some images in our database intensity inhomogeneities could also be perceived in the direction orthogonal to the anode-cathode axis. Moreover, intensity inhomogeneities may also originate from other sources, such as non-uniformity of the recording device. Future work includes extension of model (1) to two-dimensions and comparison of this correction method with other intensity correction techniques.

\section{Acknowledgments}

This work was partly supported by a grant of the Research Fund KU Leuven GOA/99/05 (Variability in Human Shape and Speech).

\section{References}

1. D. H. Ballard. Generalizing the hough transform to detect arbitrary shapes. Pattern Recognition, 13:111-122, 1981.

2. G. Behiels, D. Vandermeulen, F. Maes, P. Suetens, and P. Dewaele. Active shape model-based segmentation of digital X-ray images. Proc. MICCAI'99, Lecture notes in computer science, vol. 1679, pp. 128-137, 1999, Springer.

3. G. Behiels, D. Vandermeulen, and P. Suetens. Statistical shape model-based segmentation of digital x-ray images. In IEEE Workshop on Mathematical Methods in Biomedical Image Analysis (MMBIA'00), pp. 61-68, 2000.

4. S.N.C. Cheng, H.-P. Chan, L.T. Niklason, and R.S. Adler. Automated segmentation of regions of interest on hand radiographs. Medical Physics, 21(8):1293-1300, 1994.

5. S.L. Fritz and W.H. Livingston. A comparison of computed and measured heel effect for various target angles. Medical Physics, 9(2):216-219, 1982.

6. P. V. C. Hough. Methods and means for recognising complex patterns. U.S. Patent $3069654,1962$.

7. C. Kimme, D. Ballard, and J. Sklansky. Finding circles by an array of accumulators. In Communications of the ACM, vol. 18, pp. 120-122, 1975.

8. G. Manos, A. Y. Cairns, I. W. Ricketts, and D. Sinclair. Automatic segmentation of hand-wrist radiographs. Image and Vision Computing, 11(2):100-111, 1993.

9. J. A. Nelder and R. Mead. A simplex method for function minimization. The Computer Journal, 8:308-313, 1965.

10. K. Van Leemput, F. Maes, D. Vandermeulen, and P. Suetens. Automated modelbased bias field correction of MR images of the brain. IEEE Trans. Medical Imaging, 18(10):885-896, 1999. 